\title{
Article
}

\section{Design and Implementation of Novel Efficient Full Adder/Subtractor Circuits Based on Quantum-Dot Cellular Automata Technology}

\author{
Mohsen Vahabi ${ }^{1}$, Pavel Lyakhov ${ }^{2, *(\mathbb{D})}$ and Ali Newaz Bahar ${ }^{3}(\mathbb{1})$ \\ 1 Department of Electrical Engineering, Islamic Azad University of Science and Research Tehran (Kerman) \\ Branch, Kerman 7718184483, Iran; mohsen.vahabi@iauk.ac.ir \\ 2 Department of Automation and Control Processes, Saint Petersburg Electrotechnical University "LETI", \\ 197376 Saint Petersburg, Russia \\ 3 Department of Information and Communication Technology (ICT), Mawlana Bhashani Science and \\ Technology University, Tangail 1902, Bangladesh; bahar.ict@mbstu.ac.bd \\ * Correspondence: ljahov@mail.ru
}

Citation: Vahabi, M.; Lyakhov, P.;

Bahar, A.N. Design and

Implementation of Novel Efficient Full Adder/Subtractor Circuits Based on Quantum-Dot Cellular Automata Technology. Appl. Sci. 2021, 11, 8717. https://doi.org/10.3390/app11188717

Academic Editor: Vladimir M. Fomin

Received: 19 July 2021

Accepted: 15 September 2021

Published: 18 September 2021

Publisher's Note: MDPI stays neutral with regard to jurisdictional claims in published maps and institutional affiliations.

Copyright: (C) 2021 by the authors. Licensee MDPI, Basel, Switzerland. This article is an open access article distributed under the terms and conditions of the Creative Commons Attribution (CC BY) license (https:// creativecommons.org/licenses/by/ $4.0 /)$.

\begin{abstract}
One of the emerging technologies at the nanoscale level is the Quantum-Dot Cellular Automata (QCA) technology, which is a potential alternative to conventional CMOS technology due to its high speed, low power consumption, low latency, and possible implementation at the atomic and molecular levels. Adders are one of the most basic digital computing circuits and one of the main building blocks of VLSI systems, such as various microprocessors and processors. Many research studies have been focusing on computable digital computing circuits. The design of a Full Adder/Subtractor (FA/S), a composite and computing circuit, performing both the addition and the subtraction processes, is of particular importance. This paper implements three new Full Adder/Subtractor circuits with the lowest number of cells, lowest area, lowest latency, and a coplanar (single-layer) circuit design, as was shown by comparing the results obtained with those of the best previous works on this topic.
\end{abstract}

Keywords: Quantum-Dot Cellular Automata (QCA); Full Adder/Subtractor (FA/S); coplanar

\section{Introduction}

The QCA technology, with its unique features such as minimal dimensions, high speed, very low latency, low power consumption, and high operating frequency [1], has attracted the attention of many researchers and scientists as a new method of communication and computation. It has introduced significant novelties in the field of computer science and logic circuits. Adders are one of the most fundamental computational circuits of digital logic and have attracted researchers' attention. Adders are one of the main building blocks of many VLSI systems, such as various microprocessors and processors. Are the new designs aiming at optimizing the relevant blocks compatible with the development of this technology? A complete Adder/Subtractor design with a simple structure and low power consumption can significantly simplify digital circuits. A Full Adder/Subtractor design should include a composite computations circuit and allow performing both addition and subtraction processes. One of the problems in creating hybrid courses is the appropriate composition of wires crossover to reduce costs.

Due to the high price and increasing circuit complexity, a multilayer crossovers design in the implementation of QCA circuits is not desirable (favorable) [2,3]. To achieve coplanar crossovers, it was suggested to rotate the QCA cells, but due to the coexistence of two types of QCA cells, this caused some problems, such as low stability and high implementation cost. Therefore, a design including this type of cells is not desirable [2,4]. The best method for designing QCA circuits is based on the use of 90-degree cells with non-adjacent clock 
phases (four clock phases) to develop the crossover wires in a single layer [2,5]. Therefore, we used these two types of crossover for this research.

For this reason, The proposed designs, due to their coplanar structure and to the fact that they do not require other layers, have a reduced number of cells, occupied area, and delay. The remainder of this article is organized as follows. Section 2 (Background) provides an overview of QCA and previous literature. Section 3 (Proposed Circuits) presents the proposed architecture of Full Adder/Subtractor circuits. In Section 4 (Guidelines Performance Evaluation), we compare the proposed designs with previous architectures. In Section 5 (Conclusion), we discuss our conclusions.

\section{Background}

\subsection{The Basis of Quantum-Dot Cellular Automata (QCA) Technology}

This technology is based on QCA cells, and the basis of the QCA cell can represent a logical bit with occupied space in the nanoscale. A QCA cell includes two electrons, and, based on the Coulombic repulsion created between two electrons, two logical values of " 0 " and " 1 " are possible. The QCA cells are square, as shown in Figure 1. Each enclosure consists of four holes. The two electrons are trapped inside and can move freely between the holes; by placing two electrons in four spots, six different states are created, which is impossible due to Coulombic repulsion forces between the electrons. As a result, to satisfy these forces, electrons are placed inside the holes with as far apart as possible, until the Coulombic repulsion law is satisfied. Depending on the location of the electrons and their diameter, two structures are created; by the establishment of two electrons in each of these two poles, two different states are created. With these two types of systems, two logical values can be obtained; we will consider one of the logical values. We attribute these two polar structures 1 and -1 to the logical values of " 1 " and " 0 ", respectively; the poles at 1 and -1 same are those of the square cells, as shown in Figure 1 [6-8].

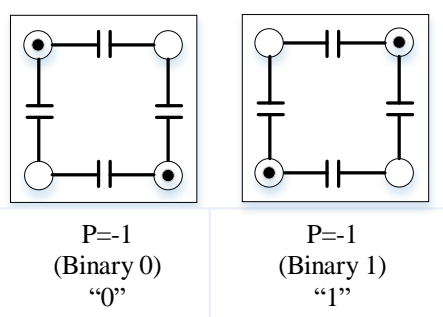

(a)

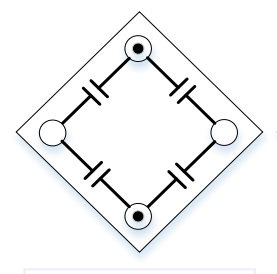

$\mathrm{P}=-1$ (Binary 0) "0"

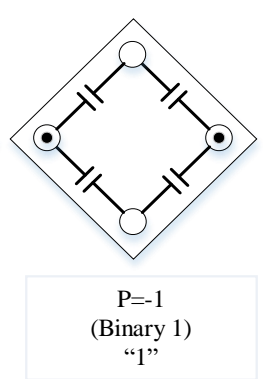

(c)

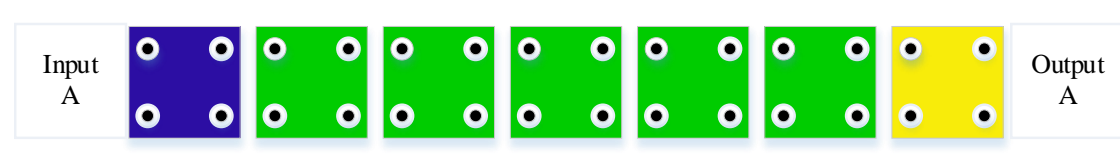

(b)

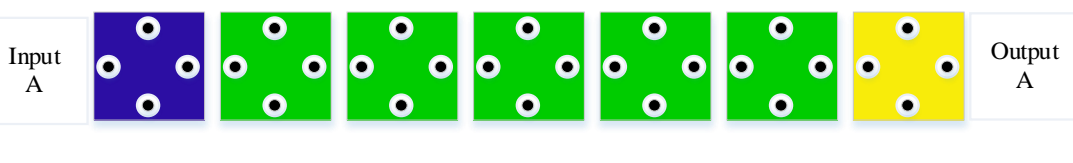

(d)

Figure 1. (a) Normal QCA cells' structure, (b) Normal QCA wire's structure, (c) Rotated QCA cells' structure and (d) Rotated QCA wire's structure.

When the electrons move inside the cell, they tunnel between the holes. Then, the moving of the electrons inside the cell is similar to a nonlinear move, and the Coulombic repulsion force is not exerted just between the electrons inside a cell. However, as shown in Figure 2, each cell adjacent to this one, which has a logical value, is affected and affects the next adjacent cell that has no value, converting it to its value [6-8]. 


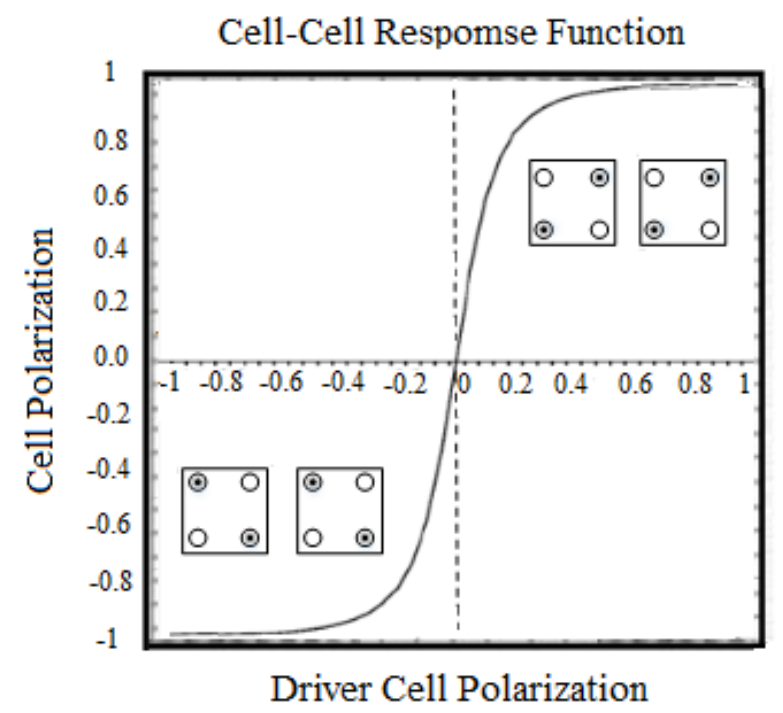

Figure 2. Behavior of adjacent cells.

\subsection{QCA Four-Phase Clock}

A four-clocking phase scheme for the QCA is shown in Figure 3. As shown in the figure, the barriers (potential barriers) rise during the first clock phase (switch). At the beginning of this phase, the borders are low, and the QCA cell is unpolarized; in this state, under the effect Columbic repulsion, the cell receives data from its adjacent cells. Then, with the barriers rising, the QCA cells are polarized according to their input drive modes, and at the end of this clock phase, the borders are high enough to prevent electron tunneling. As a result, the cell is locked. It is in this phase that the actual switching happens. During the second phase of the clock (hold), the barriers remain high. In this phase, the cell is relatively stable and transmits its data to the adjacent cells. The walls gradually decrease during the third clock phase (release), and the cell becomes unstable. In this phase, the cell is allowed to lose its polarization (unpolarized). During the fourth clock phase (relax phase), the cell barriers are in the lowest state, and the cells remain unpolarized. The cell is not used in this phase. After the end of this phase, the cell enters the switch phase again [3,9].

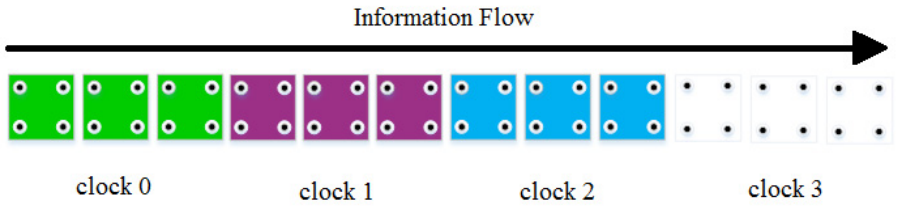

(a)

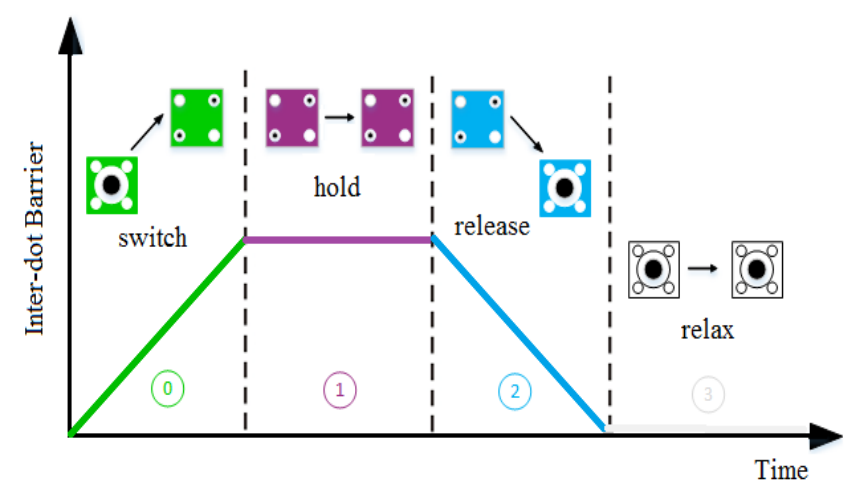

(b)

Figure 3. (a) Clock phases and (b) QCA four-phase clock mechanism. 


\subsection{QCA Four-Phase Clock}

One of the gates used in logic circuits is the inverter gate (Not gate). A type of inverter gate used in QCA technology is shown in Figure 4. It is used for inverting the desired signal as required $[10,11]$.

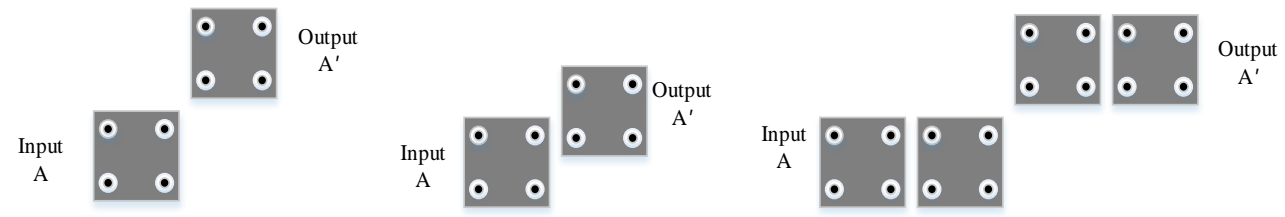

Figure 4. Two different Not-gate in QCA based on $90^{\circ}$ cells.

One of the most usable logic gates in QCA technology is the majority gate. This gate has an odd number of inputs and one output. In other words, the output cell value (output cell polarization) is determined according to the logical value of the majority inputs. As a result, the output cell value is determined based on the majority of inputs $[8,10]$. Figure 5a shows an example of this gate.

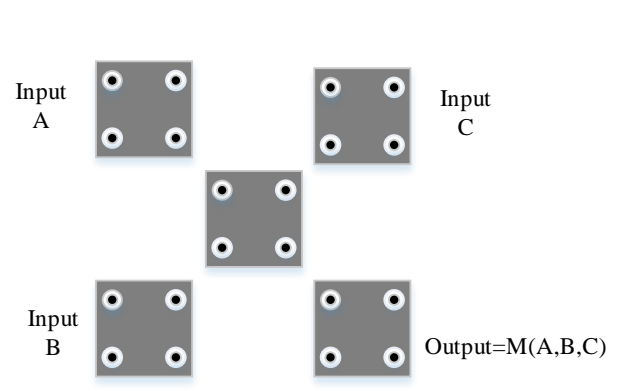

(a)

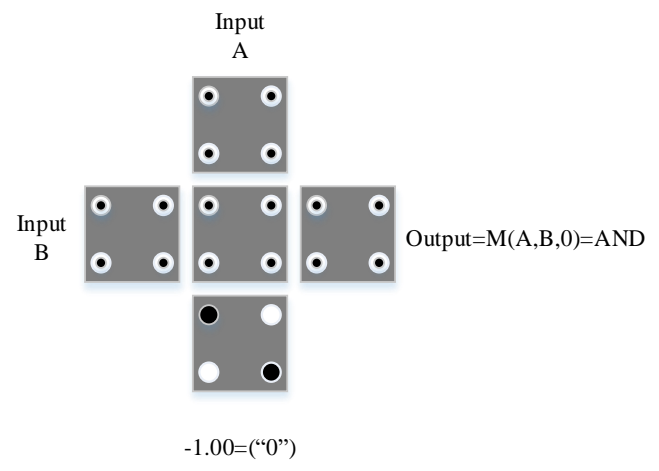

(b)
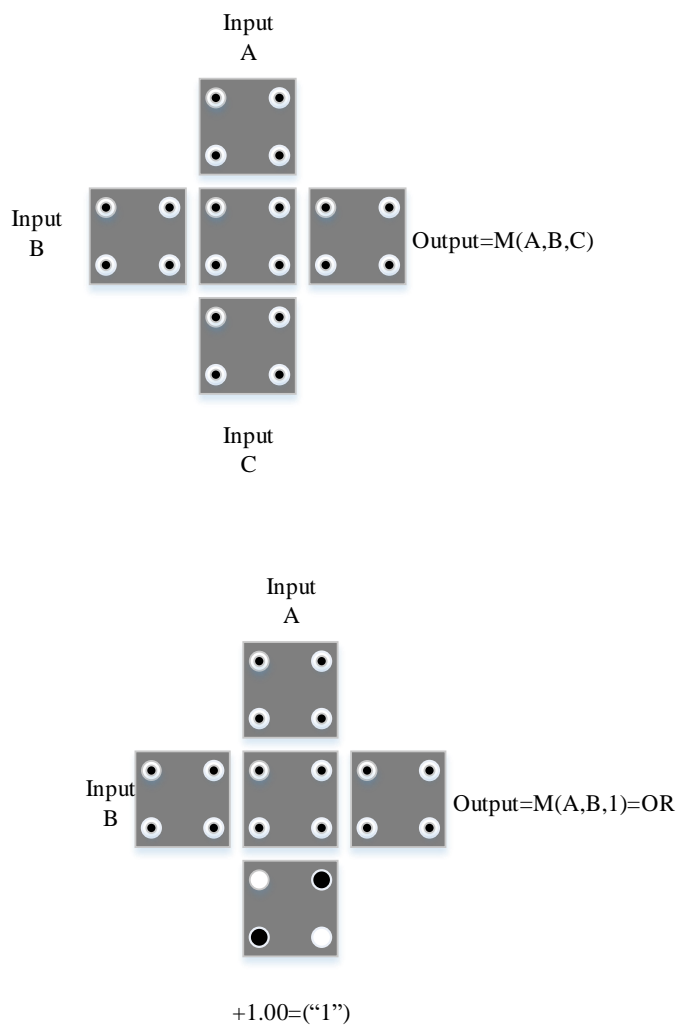

(c)

Figure 5. (a) QCA with implementation of the majority gate, (b) QCA with implementation of the AND Gate with two inputs and (c) QCA with implementation of the OR Gate with two inputs.

By stabilizing (fixed) one of the inputs of the majority gate and considering a logical value " 0 " (polarization -1 ), the AND gate is generated [10,12]. Figure $5 b$ shows a two-input AND gate.

The OR gate is generated by fixing one of the majority gate inputs and considering a logical value "1" (polarization +1 ) $[10,12]$. Figure 5 c shows a two-input OR gate. 


\subsection{Related Work}

In a previous paper [13], the architecture of a Full Adder/Full Subtractor with a multilayer crossover design was described (Figure 6). This type of multilayer design requires a larger consumption area than we planned. It has also more cells and delays with respect to our designs, so its cost is very high. As a result, our proposed technique was implemented using the coplanar method. It has significant advantages over this type of architecture regarding cell number, delay, and area consumption.

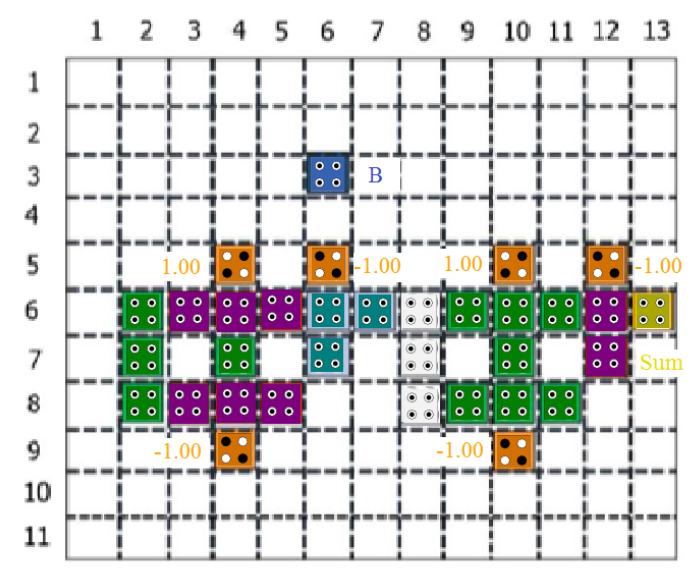

Layer 1

(a)

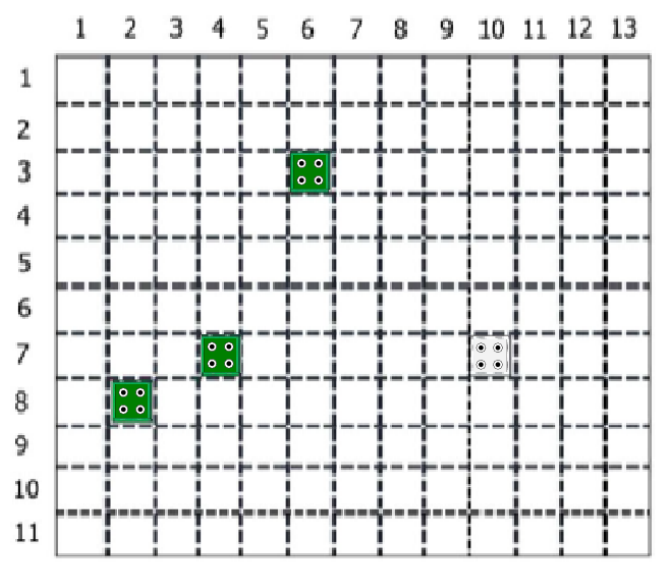

Layer 2

(b)

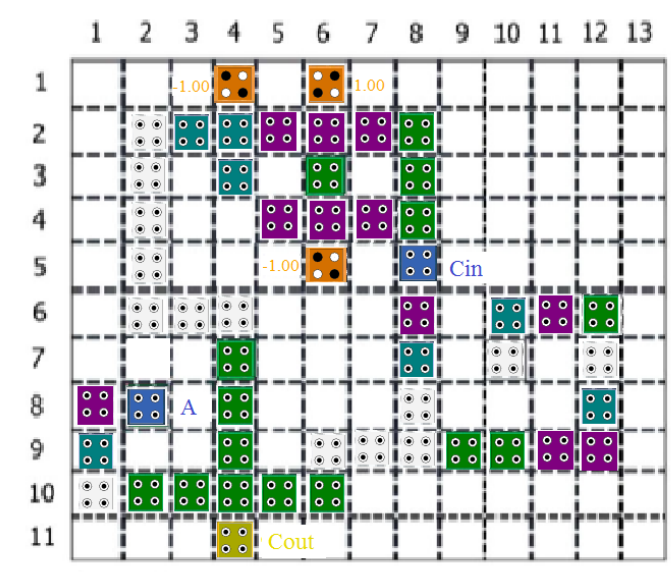

Layer 3

(c)

Figure 6. Full Adder/Full Subtractor circuit [13].

In another paper [14], the architecture of a Full Adder/Full Subtractor was also presented (Figure 7). This type of design, due to the high delay and unsuitable carry output, requires another crossover. That leads to an increase in the delay and number of cells. As a result, this architecture is also not suitable. Our proposed design has significant advantages relative to this design [14], such as the number of cells, delay, area of consumption, and therefore cost function. 


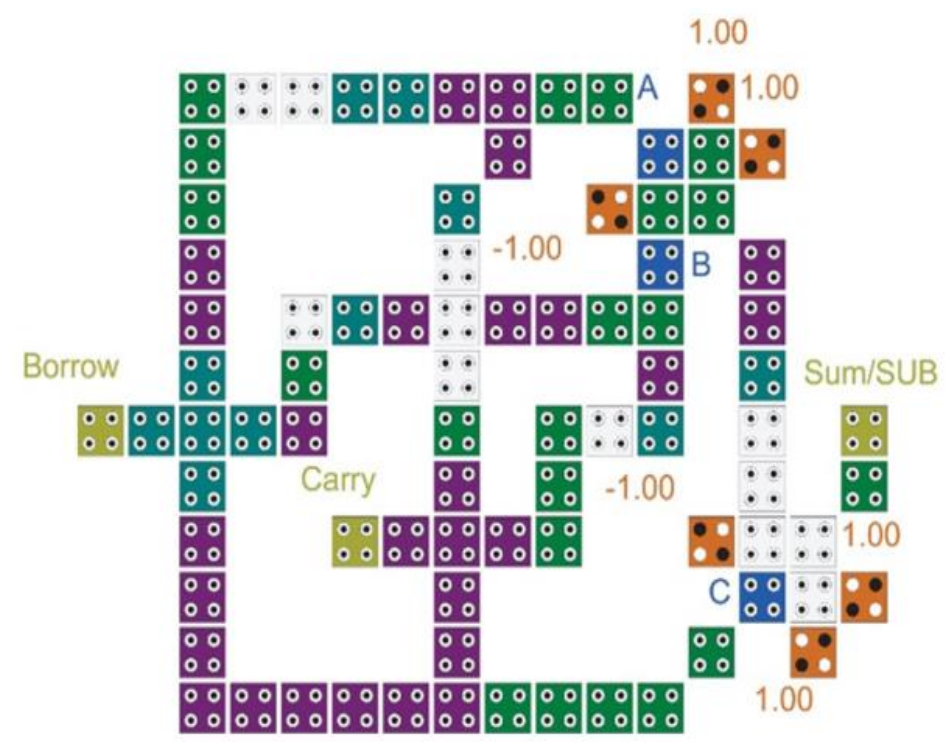

Figure 7. Full Adder/Full Subtractor circuit [14].

Another paper [15] described a Full Adder/Full Subtractor architecture using the coplanar method design (Figure 8 ). This type of design is not very favorable, because the rotated cells $\left(45^{\circ}\right.$ cells $)$ make it more vulnerable and increase the implementation costs. Our method has significant advantages also relative to this design [15], such as the number of cells, delay, and area. Besides, normal cells were used. Our design (C) is 50\% better than that design.

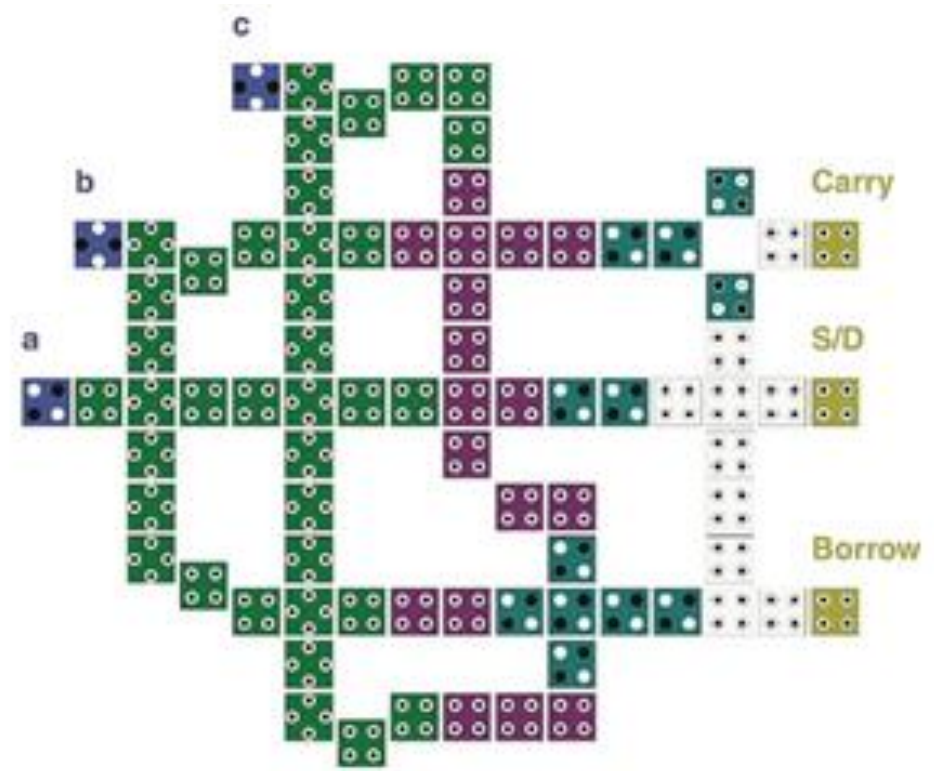

Figure 8. Full Adder/Full Subtractor circuit [15].

In a paper [16], a Full Adder/Full Subtractor circuit architecture, implemented in a coplanar method design was presented. Our proposed designs (A and B) are an addition to the coplanar designs in terms of number cells, area and, therefore, cost of implementation and have significant advantages relative to this design (Figure 9). Despite not using rotated cells in our designs (A and B), their latency is equal to that reported in this previous article. In comparison, our third design delay (C) is $33.34 \%$ superior to this design and is ideal in terms of cell number and area. 


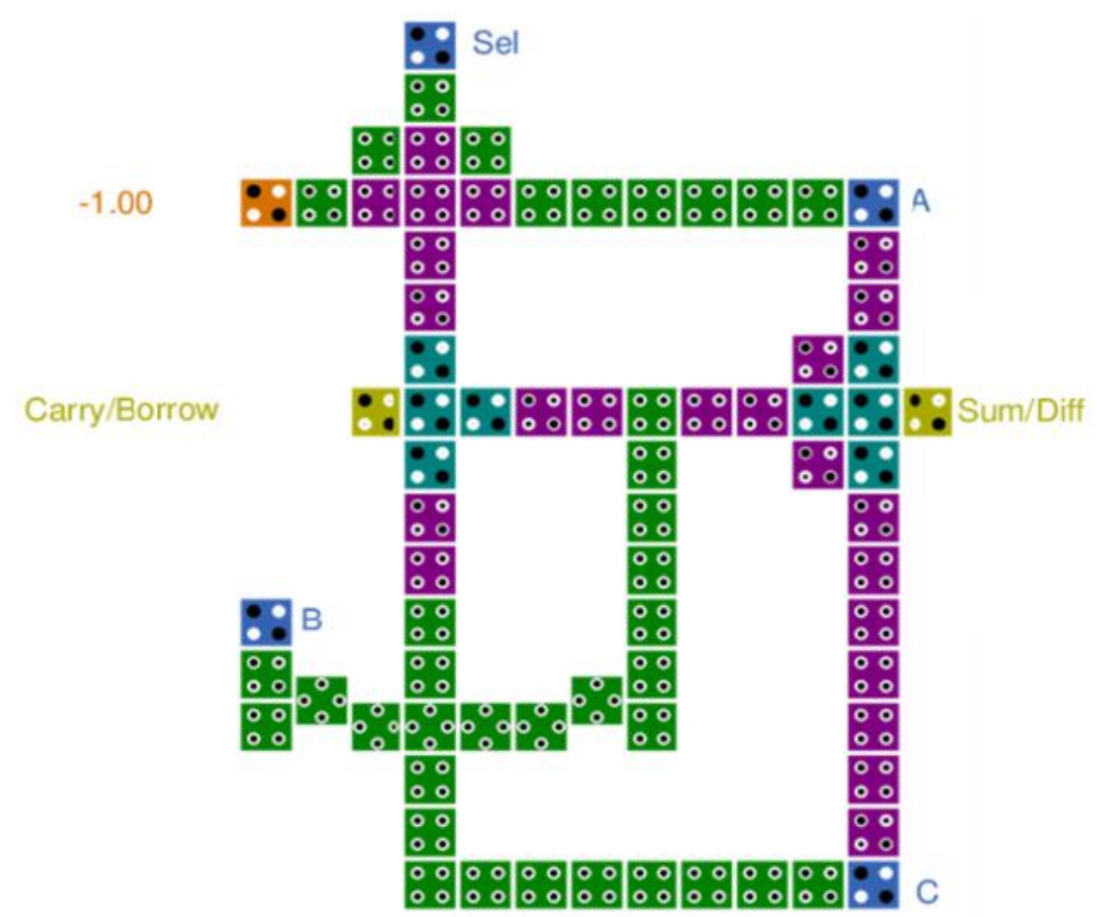

Figure 9. Full Adder/Full Subtractor circuit [16].

Another paper [17] also presented two Full Adder/Full Subtractor circuit designs, as shown in Figures 10 and 11; these designs are also coplanar, but our designs have significant advantages in terms of number of cells, delay, and area.

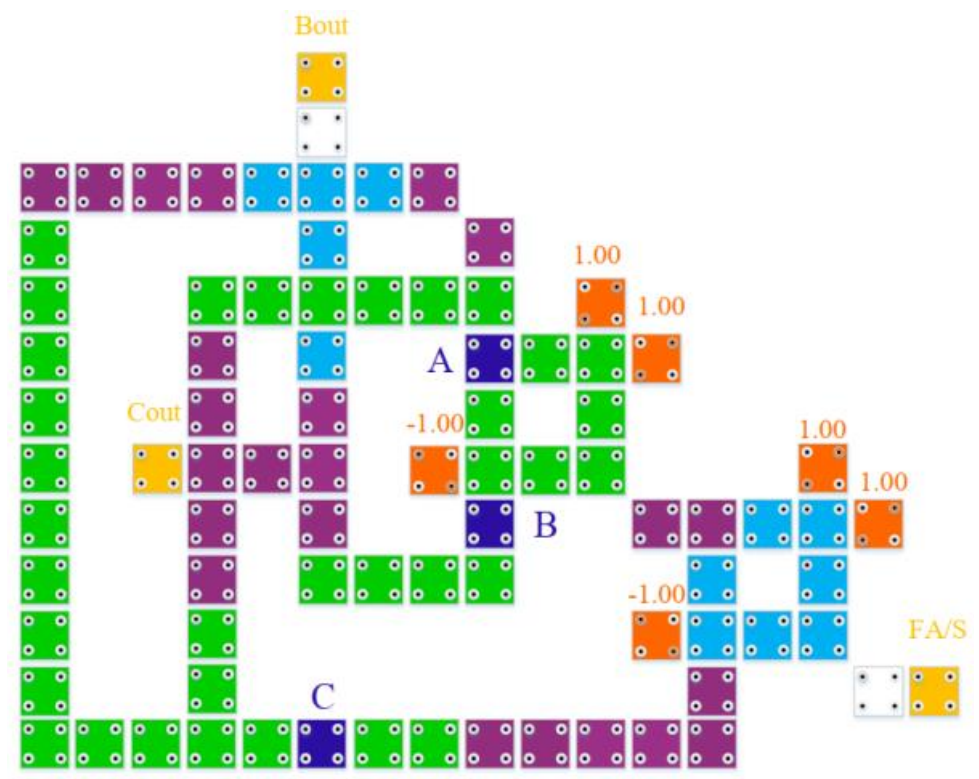

Figure 10. Full Adder/Full Subtractor circuit [17]-a. 


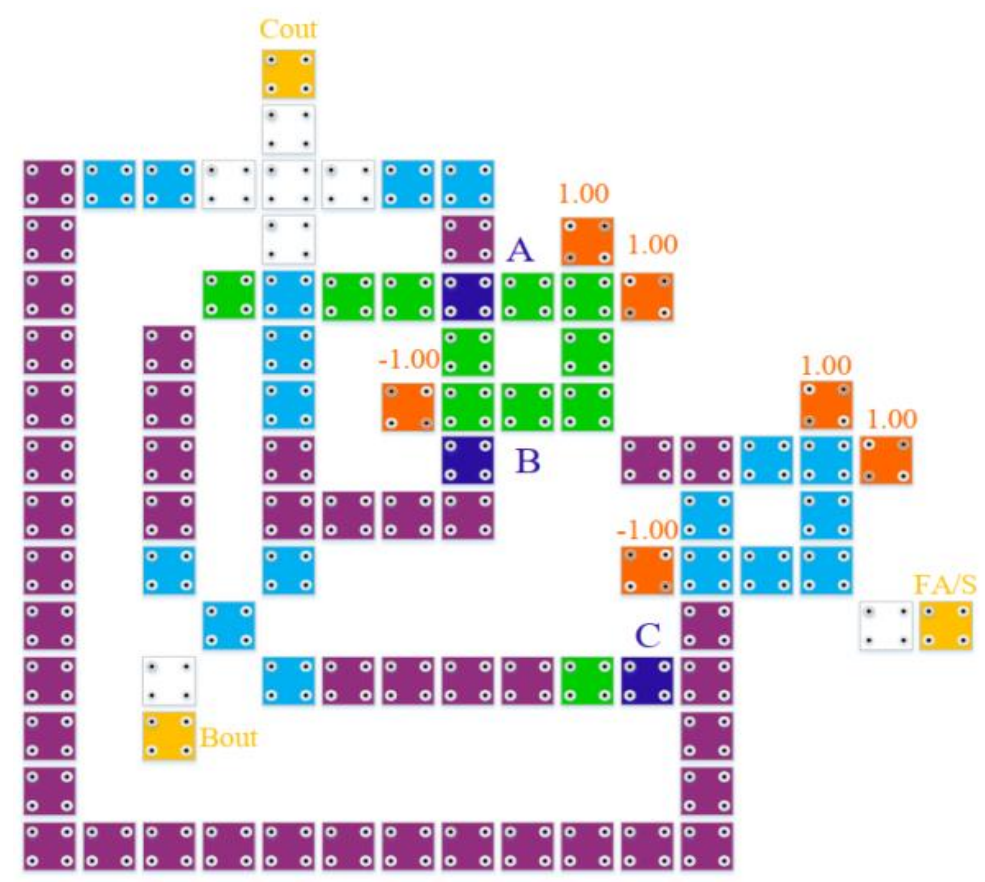

Figure 11. Full Adder/Full Subtractor circuit [17]-b.

\section{The Proposed Circuits}

In this paper, we designed three new Full Adder/Subtractor (FA/S) circuits based on the XOR gate [18] with the lowest number of cells, smallest consumption circuit area, and lowest latency (delay) relative to the previous best circuits. In cases, a single-layer (coplanar) design was used. Therefore, these circuits are the best examples of design ever made. The two designs (A and B) are coplanar and use only standard cells ( $90^{\circ}$ cells). In the third design (C) which is coplanar, the cells are rotated $\left(45^{\circ}\right.$ cells) and also coplanar. The third design shows that the use of this type of cell may reduce the delay of circuit outputs, but, it also reduces the stability and resistance of the circuit compared to the circuits with standard cells.

\subsection{FA/S Circuits Design}

A Full Adder/Subtractor circuit is a combination circuit where two addition and subtraction operations are performed. This circuit has three inputs (A, B, Cin) and three outputs ( $\backslash \backslash D$, Cout, Bout) $[19,20]$. Equation (1) is the equation of the output $S \backslash D$, Equation (2) is the equation of the Cout output, and Equation (3) is the equation of the Bout output. Figure 12, block diagram, and Table 1 show the correct Table of this circuit.

$$
\begin{gathered}
\mathrm{S} \backslash \mathrm{D}=\mathrm{A} \oplus \mathrm{B} \oplus \mathrm{C} \text { in } \\
\text { Cout }=\mathrm{M}(\mathrm{A}, \mathrm{B}, \mathrm{Cin})=\mathrm{A} \cdot \mathrm{B}+\mathrm{A} \cdot \mathrm{C}+\mathrm{B} \cdot \mathrm{C} \\
\text { Bout }=\mathrm{M}\left(\mathrm{A}^{\prime}, \mathrm{B}, \mathrm{Cin}\right)=\mathrm{A}^{\prime} \cdot \mathrm{B}+\mathrm{A}^{\prime} \cdot \mathrm{C}+\mathrm{B} \cdot \mathrm{C}
\end{gathered}
$$

This paper designed FA/S circuits with the lowest number of cells, lowest consumable area, and lowest latency (delay), compared to the previous best examples. We used a singlelayer (coplanar) design to obtain the best designs ever made. The design is better than previous ones not only in terms of cell number, area, and delay but also because it is based on a single layer. Figure 13 presents a block diagram of these circuits and Figures 14-16, show the implementation of the Proposed Full Adder/Subtractor (FA/S) circuits designs. 


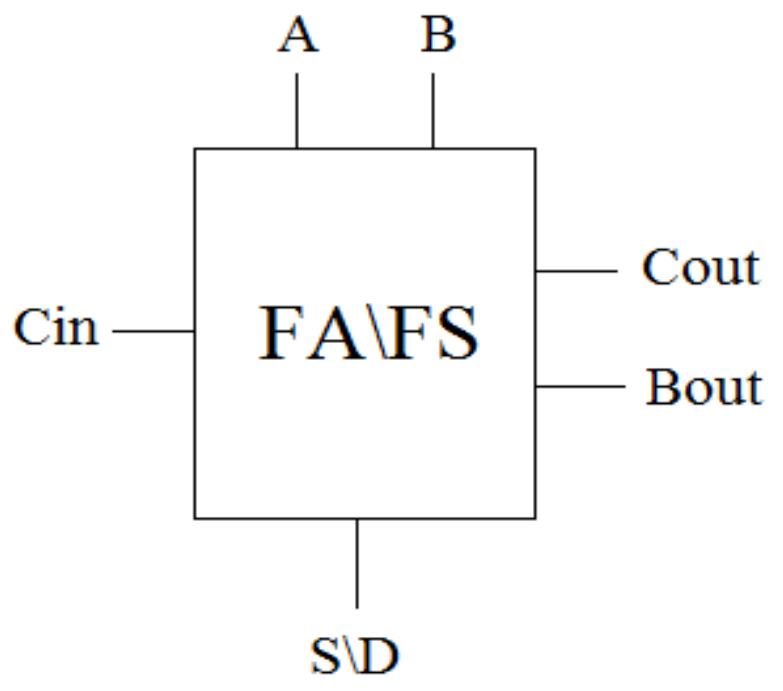

Figure 12. Block diagram of the Full Adder/Subtractor circuit.

Table 1. Truth table of the Full Adder/Subtractor.

\begin{tabular}{cccccc}
\hline Bout & Cout & $\mathbf{S} \backslash \mathbf{D}$ & Cin & B & A \\
\hline 0 & 0 & 0 & 0 & 0 & 0 \\
1 & 0 & 1 & 1 & 0 & 0 \\
1 & 0 & 1 & 0 & 1 & 0 \\
1 & 1 & 0 & 1 & 1 & 0 \\
0 & 0 & 1 & 0 & 0 & 1 \\
0 & 1 & 0 & 1 & 0 & 1 \\
0 & 1 & 0 & 0 & 1 & 1 \\
1 & 1 & 1 & 1 & 1 & 1 \\
\hline
\end{tabular}

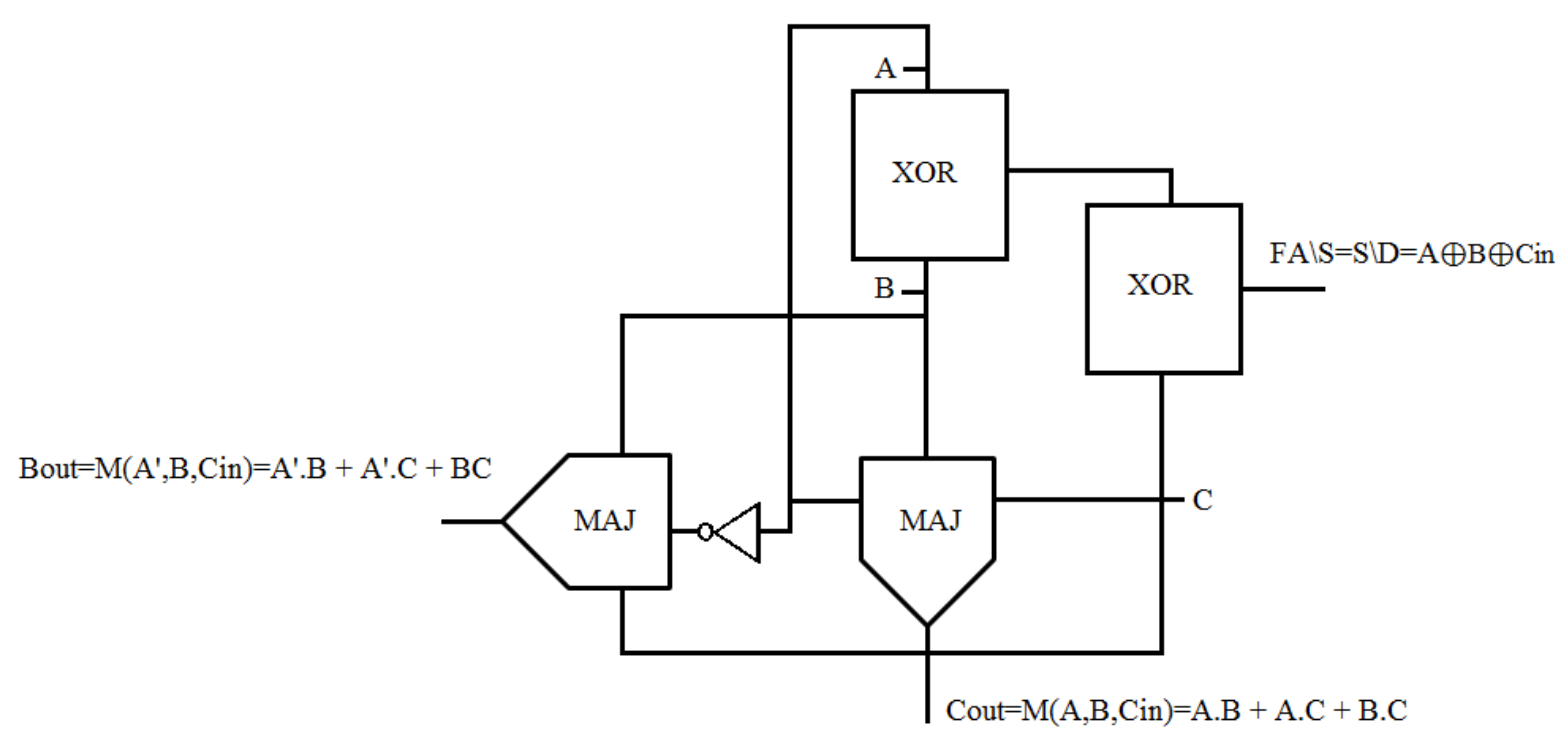

Figure 13. Simulation results of the proposed XOR-gate. 


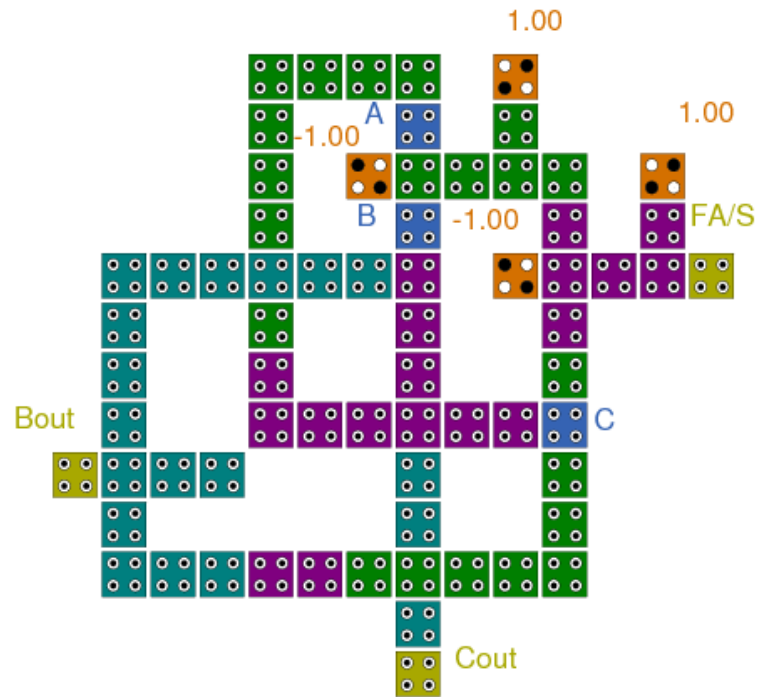

Figure 14. The proposed (A) Full Adder/Full Subtractor circuit.

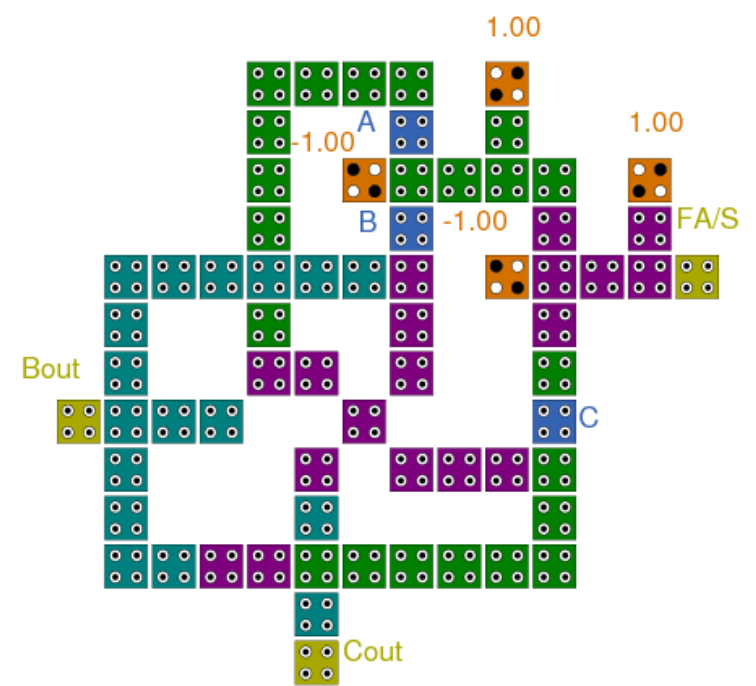

Figure 15. The proposed (B) Full Adder/Full Subtractor circuit.

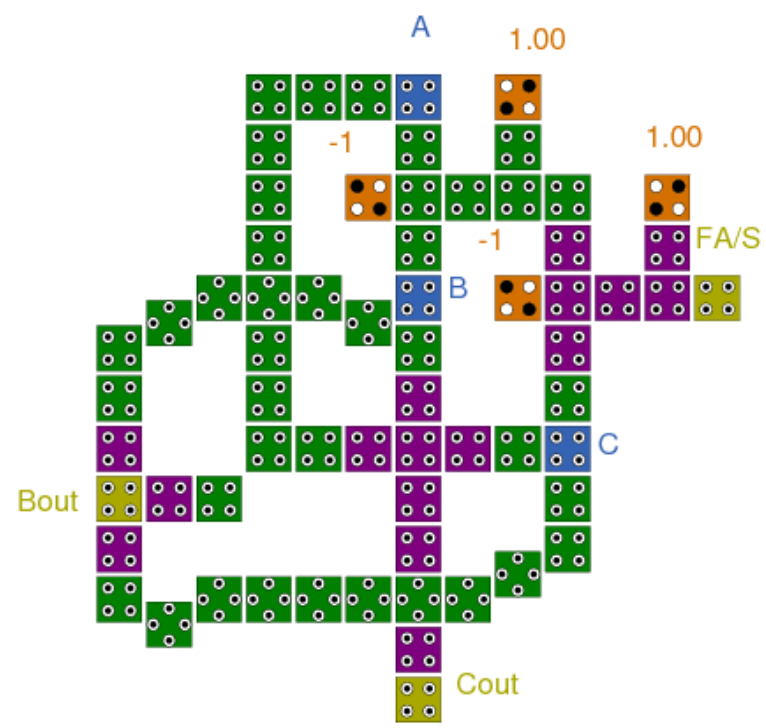

Figure 16. The proposed (C) Full Adder/Full Subtractor circuit. 


\subsection{Simulation Results}

In this section, the simulator outputs of the proposed circuits are shown in Figures 17-19. The output latency of both offered courses (A and B) is the same, and they provide the same simulation outputs. As can be seen, in both circuits, the delay is one clock (four phases). The delay of the third circuit (C) is 0.5 clock (two stages). The proposed Full Adder/Subtractor hybrid circuits combine two addition and subtraction circuits and allow the concurrent performance of both operations.

\begin{tabular}{|l|}
\hline max: $1.00 e+000$ \\
min: $-1.00 e+000$ \\
\hline max: $1.00 e+000$ \\
B \\
min: $-1.00 e+000$ \\
\hline max: $1.00 e+000$ \\
C \\
min: $-1.00 e+000$ \\
\hline max: $9.30 e-001$ \\
FAVS \\
min: $-9.65 e-001$ \\
\hline max: $9.51 e-001$ \\
Cout \\
min: $-9.51 e-001$ \\
\hline max: $9.44 e-001$ \\
Bout \\
min: $-9.44 e-001$ \\
\hline max: $9.80 e-022$ \\
CLOCK 1 \\
min: $3.80 e-023$ \\
\hline max: $9.80 e-022$ \\
CLOCK 2 \\
min: $3.80 e-023$ \\
\hline
\end{tabular}
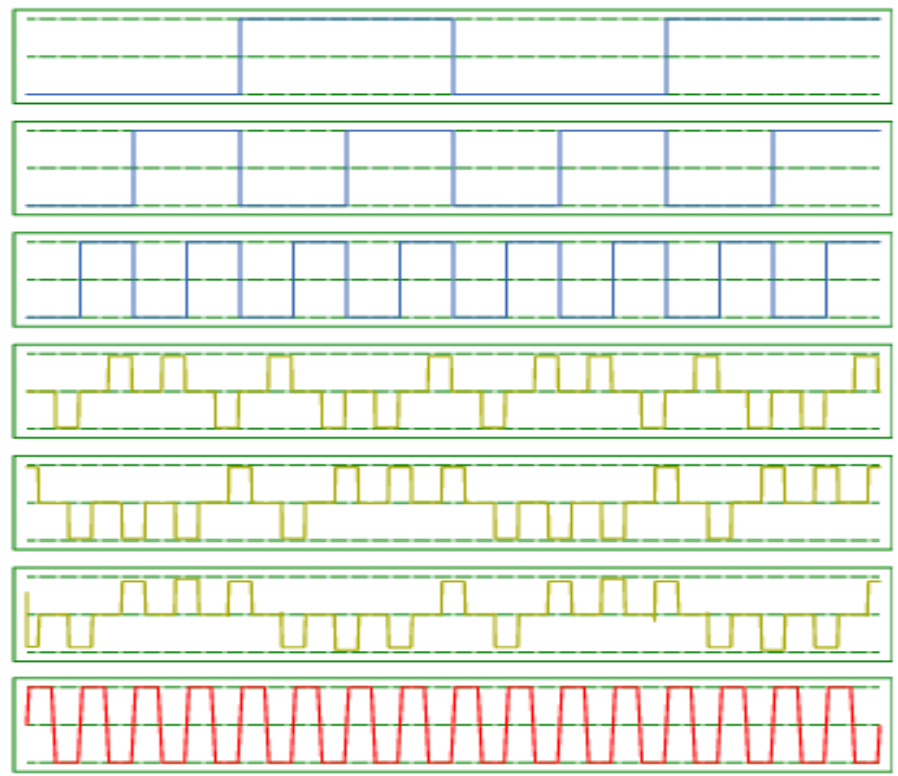

CLOCK 2

min: $3.80 e-023$

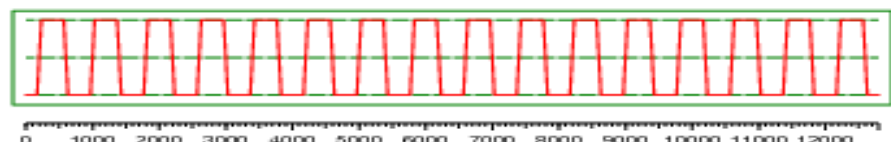

Figure 17. Simulation results for the proposed (A) Full Adder/Full Subtractor circuit.

\begin{tabular}{l}
\hline max: $1.00 e+000$ \\
min: $-1.00 e+000$ \\
\hline max: $1.00 e+000$ \\
B \\
min: $-1.00 e+000$ \\
\hline max: $1.00 e+000$ \\
C \\
min: $-1.00 e+000$ \\
\hline max: $9.30 e-001$ \\
FAvS \\
min: $-9.65 e-001$ \\
\hline max: $9.52 e-001$ \\
Cout \\
min: $-9.52 e-001$ \\
\hline max: $9.20 e-001$ \\
Bout \\
min: $-8.80 e-001$ \\
\hline max: $9.80 e-022$ \\
CLOCK 1 \\
min: $3.80 e-023$ \\
\hline max: $9.80 e-022$ \\
CLOCK 2 \\
min: $3.80 e-023$ \\
\hline
\end{tabular}
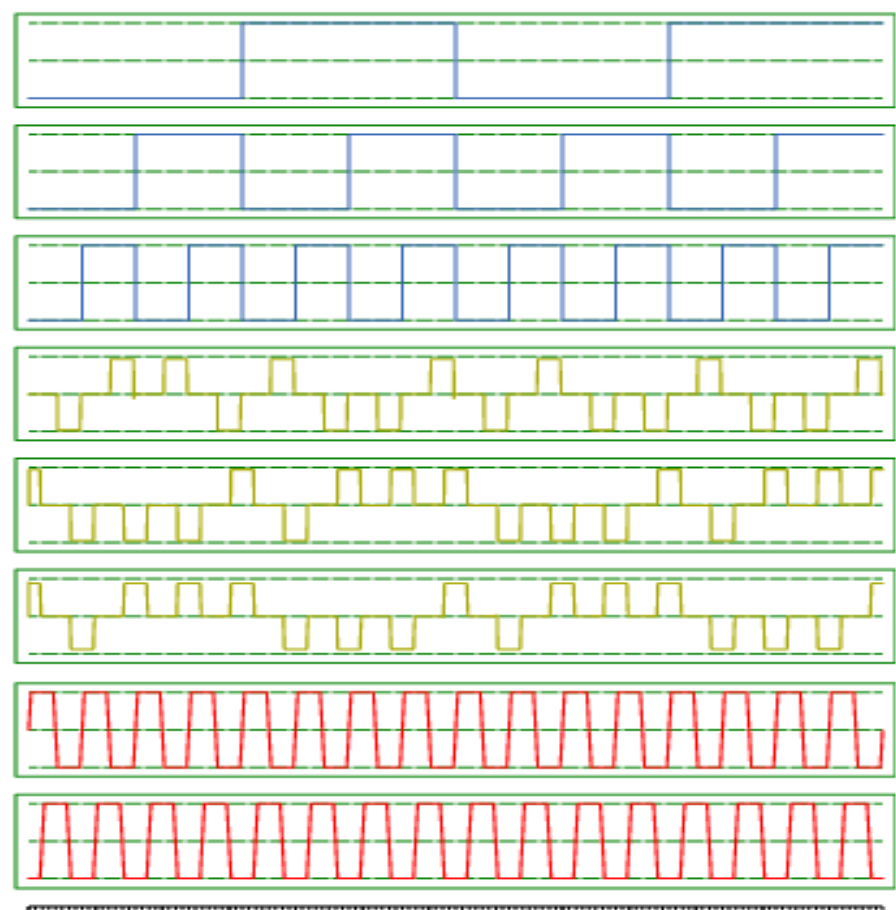

Figure 18. Simulation results for the proposed (B) Full Adder/Full Subtractor circuit. 
max: $1.00 e+000$

A

min: $-1.00 e+000$

max: $1.00 e+000$

B

min: $-1.00 e+000$

max: $1.00 e+000$

C

min: $-1.00 e+000$

max: $9.30 e-001$

FAVS

min: $-9.65 e-001$

max: $9.49 e-001$

min: $-9.49 e-001$

max: 9.94e-001

Bout

min: $-9.94 e-001$

max: $9.80 e-022$

CLOCK 1

min: 3.80 e-023
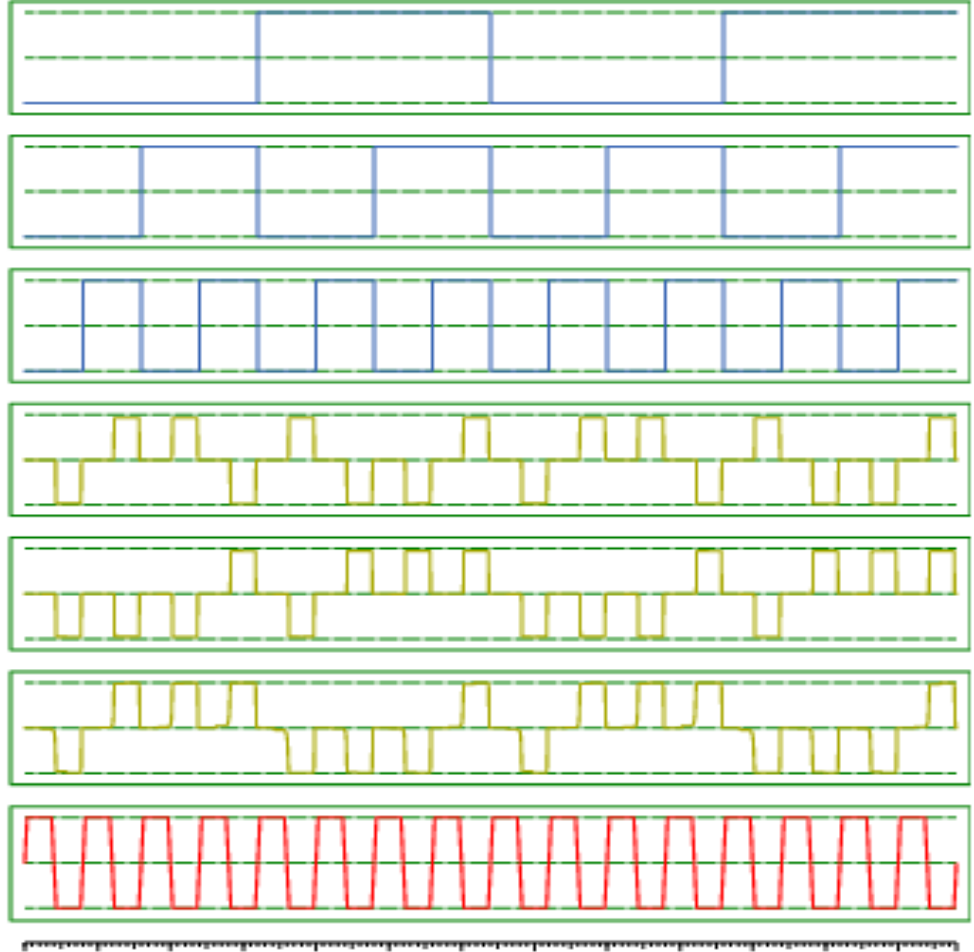

D $100020003000400050006000 \quad 7000$ 9000 9000 10000 1100012000

Figure 19. Simulation results for the proposed (C) Full Adder/Full Subtractor circuit.

\section{Guidelines of Performance Evaluation}

The QCA Designer provided the simulation results. The simulation parameters are presented in Table 2. The proposed design was compared with designs described in previous works. For all circuits designed, parameters including area, delay, and cell numbers are provided. The type of crossover is also presented for a better and more accurate comparison.

Table 2. Simulation parameters for the QCA Designer.

\begin{tabular}{cc}
\hline Parameter & Value \\
\hline Cell width & $18 \mathrm{~nm}$ \\
Cell height & $18 \mathrm{~nm}$ \\
Dot diameter & $5 \mathrm{~nm}$ \\
Number of samples & 12,800 \\
Convergence tolerance & 0.001 \\
Radius of effect & $65 \mathrm{~nm}$ \\
Relative permittivity & 12.9 \\
Clock high & $9.8 \times 10^{-22} \mathrm{~J}$ \\
Clock low & $3.8 \times 10^{-23} \mathrm{~J}$ \\
Clock amplitude factor & 2 \\
Layer separation & $11.5 \mathrm{~nm}$ \\
Maximum iteration per sample & 100 \\
\hline
\end{tabular}

The simulation results are given in Table 3. As can be seen, the proposed circuits were compared with the best circuits previously described. In Table 3, consumption area, delay, and cell number of the proposed Full Adder/Subtractor circuits are compared to those of previous designs. 
Table 3. Comparing the Full Adder/Subtractor (FA/S) of this study with those of previous works.

\begin{tabular}{ccccc}
\hline Crossover Type & $\begin{array}{c}\text { Latency } \\
\text { (clock) }\end{array}$ & Cell Count & $\begin{array}{c}\text { Area } \\
\left(\boldsymbol{\mu m}^{\mathbf{2}}\right)\end{array}$ & Circuit \\
\hline Multi-Layer & 1.5 & 90 & 0.6 & {$[13]$} \\
Coplanar (clocking based) & 1.5 & 83 & 0.09 & {$[14]$} \\
Coplanar (rotated cells) & 1 & 82 & 0.11 & {$[15]$} \\
Coplanar (rotated cells) & 0.75 & 75 & 0.09 & {$[16]$} \\
Coplanar (clocking based) & 1 & 92 & 0.09 & {$[17]-\mathrm{a}$} \\
Coplanar (clocking based) & 1 & 84 & 0.09 & {$[17]-\mathrm{b}$} \\
Coplanar (clocking based) & 0.75 & 68 & 0.072 & Proposed A \\
Coplanar (clocking based) & 0.75 & 67 & 0.072 & Proposed B \\
Coplanar (rotated cells) & 0.5 & 65 & 0.067 & Proposed C \\
\hline
\end{tabular}

As shown in Table 3, our designs (A) and (B) allow reducing the area and power consumption up to $39.1 \%$ with respect to previous circuits described in [14,17]. As can be seen, the delay in the proposed designs improved significantly with respect to previous works. Our designs (A) and (B) reduce the delay by $50 \%$ in comparison to the designs in $[13,14]$ and by $30 \%$ with respect to those in $[15,17]$. The reduction in the proposed design C, relative to the designs in $[13,14]$, corresponds to $66.66 \%$, whereas it corresponds to $50 \%$ in comparison to those in [15], [17]-a and [17]-b, and to $33.33 \%$ in comparison to that in [16]. As can be seen, the proposed designs have also the lowest cell number with respect to the other designs. Improvement in the cell number of the proposed design A relative to the designs in [13-17]-a and [17]-b is about $24.45 \%, 18.07 \%, 17.07 \%, 9.34 \%, 26.09 \%$, and $19.05 \%$ respectively; the cell number improvement in the proposed design B relative to [13-17]-a and [17]-b, respectively, is about $25.56 \%, 19.28 \%, 18.29 \%, 10.67 \%, 27.17 \%$, and $20.24 \%$; finally, the cell number improvement in the proposed design C relative to [13-17]-a and [17]-b, respectively, is about $27.78 \%, 21.69 \%, 20.73 \%, 13.34 \%, 29.35 \%$, and $22.62 \%$.

\section{Conclusions}

The FA/S designs using the QCA technology use at least three layers for the crossover, while several techniques use $45^{\circ}$ cells. Indeed, only non-adjacent clock phases (four clock phases) are required to design the crossover in a single layer, which is robust and better. However, the coplanar crossover's design using rotated cells can reduce delay in the circuit. In some cases, depending on the type of usage, these two types of design can be used. The circuits' designs proposed in this study are better and preferable than previous designs in terms of number of cells consumed, circuit area, delay, and cost. As a result, these three proposed designs can be used in more extensive and more complex circuits.

Author Contributions: Conceptualization, M.V.; methodology, M.V.; validation, M.V., P.L. and A.N.B.; formal analysis, M.V.; investigation, M.V., P.L. and A.N.B.; writing—original draft preparation, M.V.; writing-review and editing, P.L. and A.N.B.; supervision, P.L. and A.N.B. All authors have read and agreed to the published version of the manuscript.

Funding: This research received no external funding.

Data Availability Statement: Data are contained within the article.

Conflicts of Interest: The authors declare no conflict of interest.

\section{References}

1. Mohammadi, Z.; Mohammadi, M. Implementing a one-bit reversible full adder using quantum-dot cellular automata. Quantum Inf. Process. 2014, 13, 2127-2147. [CrossRef]

2. Tougaw, P.D.; Lent, C.S. Logical devices implemented using quantum cellular automata. J. Appl. Phys. 1994, 75, 1818-1825. [CrossRef]

3. Cho, H.; Swartzlander, E.E. Adder Designs and Analyses for Quantum-Dot Cellular Automata. IEEE Trans. Nanotechnol. 2007, 6, 374-383. [CrossRef] 
4. Huang, J.; Momenzadeh, M.; Tahoori, M.B.; Lombardi, F. Design and characterization of an and-or-inverter (AOI) gate for QCA implementation. In Proceedings of the 14th ACM Great Lakes Symposium on VLSI 2004, Boston, MA, USA, 26-28 April 2004; pp. 426-429. [CrossRef]

5. Abedi, D.; Jaberipur, G.; Sangsefidi, M. Coplanar full adder in quantum-dot cellular automata via clock-zone-based crossover. IEEE Trans. Nanotechnol. 2015, 14, 497-504. [CrossRef]

6. Fortes, J.A. Future challenges in VLSI system design. In Proceedings of the IEEE Computer Society Annual Symposium on VLSI, Tampa, FL, USA, 20-21 February 2003; pp. 5-7.

7. Frost, S.E.; Rodrigues, A.F.; Janiszewski, A.W.; Rausch, R.T.; Kogge, P.M. Memory in motion: A study of storage structures in QCA. In Proceedings of the First Workshop on Non-Silicon Computing, Cambridge, MA, USA, 3 February 2002 ; Volume 2.

8. Wang, W.; Walus, K.; Jullien, G.A. Quantum-dot cellular automata adders. In Proceedings of the 2003 Third IEEE Conference on Nanotechnology, San Francisco, CA, USA, 12-14 August 2003; Volume 1, pp. 461-464.

9. Kianpour, M.; Sabbaghi-Nadooshan, R. Optimized Design of Multiplexor by Quantum-dot Cellular Automata. Int. J. Nanosci. Nanotechnol. 2013, 9, 15-24.

10. Beigh, M.R.; Mustafa, M.; Ahmad, F. Performance Evaluation of Efficient XOR Structures in Quantum-Dot Cellular Automata (QCA). Circuits Syst. 2013, 4, 147-156. [CrossRef]

11. Swartzlander, E.E.; Cho, H.; Kong, I.; Kim, S.W. Computer arithmetic implemented with QCA: A progress report. In Proceedings of the 2010 Conference Record of the Forty Fourth Asilomar Conference on Signals, Systems and Computers, Pacific Grove, CA, USA, 7-10 November 2010; pp. 1392-1398.

12. Modi, S.; Tomar, A.S. Logic gate implementations for quantum dot cellular automata. In Proceedings of the 2010 International Conference on Computational Intelligence and Communication Networks, Bhopal, India, 26-28 November 2010.

13. Barughi, Y.Z.; Heikalabad, S.R. A Three-Layer Full Adder/Subtractor Structure in Quantum-Dot Cellular Automata. Int. J. Theor. Phys. 2017, 56, 2848-2858. [CrossRef]

14. Zoka, S.; Gholami, M. A novel efficient full adder-subtractor in QCA nanotechnology. Int. Nano Lett. 2018, 9, 51-54. [CrossRef]

15. Sadeghi, M.; Navi, K.; Dolatshahi, M. Novel efficient full adder and full subtractor designs in quantum cellular automata. J. Supercomput. 2019, 76, 2191-2205. [CrossRef]

16. Raj, M.; Gopalakrishnan, L.; Ko, S.-B. Design and analysis of novel QCA full adder-subtractor. Int. J. Electron. Lett. 2020, 9, 1-14. [CrossRef]

17. Vahabi, M.; Sabbagh Molahosseini, A. A New Coplanar Full Adder/Subtractor in Quantum-Dot Cellular Automata Technology. Majlesi J. Telecommun. Devices 2018, 7, 53-63.

18. Chabi, A.M.; Roohi, A.; Khademolhosseini, H.; Sheikhfaal, S.; Angizi, S.; Navi, K.; DeMara, R.F. Towards ultra-efficient QCA reversible circuits. Microprocess. Microsyst. 2017, 49, 127-138. [CrossRef]

19. Gladshtein, M. Design and simulation of novel adder/subtractors on quantum-dot cellular automata: Radical departure from Boolean logic circuits. Microelectron. J. 2013, 44, 545-552. [CrossRef]

20. Ahmad, F.; Ahmed, S.; Kakkar, V.; Bhat, G.M.; Bahar, A.N.; Wani, S. Modular design of ultra-efficient reversible full addersubtractor in QCA with power dissipation analysis. Int. J. Theor. Phys. 2018, 57, 2863-2880. [CrossRef] 